ISSN: 0212-5374

DOI: http://dx.doi.org/10.14201/et2014321123145

\title{
UTILIZACIÓN DIDÁCTICA DEL CINE EN MATEMÁTICAS
}

\author{
Didactic use of cinema in Mathematics
}

\section{Usage didactique du cinéma en Mathématiques}

\author{
Pablo Beltrán Pellicer* y Anna Asti** \\ * Profesor de Matemáticas de Secundaria en Aragón. Máster en Innovación e \\ Investigación en Educación (UNED). Correo-e: pablo.beltran.pellicer@gmail.com \\ ** Profesora de Matemáticas de Secundaria en Italia. Unità Città Studi del Centro \\ "Matematita". Correo-e: annaastimariani@gmail.com
}

Recibido: 02.05.2013; Aceptado: 24.11.2013; Publicado: 31.10.2014 BIBLID [0212-5374 (2014) 32, 2; 123-145]

Ref. Bibl. PABLO BELTRÁN PELLICER y ANNA ASTI. Utilización didáctica del cine en matemáticas. Enseñanza \& Teaching, 32, 2-2014, 123-145.

RESUMEN: Diversos autores y profesores se han interesado en los últimos años por el uso del cine y las series de TV como recurso didáctico en Matemáticas en educación secundaria, debido principalmente a la motivación que este medio produce en los alumnos. A este respecto, se han elaborado sugerentes recopilaciones de escenas con referencias matemáticas, análisis detallados de películas con fuerte relación con las matemáticas e, incluso, materiales didácticos para ser empleados en clase. El presente artículo propone un marco teórico inicial para el diseño de secuencias de aula basadas en las situaciones didácticas que pueden generarse a partir de fragmentos de películas o series. Para ello, se sigue un proceso de ingeniería didáctica en el que se tienen en cuenta ciertas peculiaridades, como la referente a la transposición didáctica, ya que se hace necesario considerar cuál es el conocimiento en juego dentro del fragmento y cómo aparece. Asimismo, se presenta una experiencia de clase diseñada según este proceso en el seno de un proyecto de colaboración entre dos centros de educación secundaria, donde se ha comprobado un aumento en la motivación de los alumnos, debido a trabajar con situaciones matemáticas del 
mundo real (o ficticio pero asimilable por ellos). También se han encontrado indicios de que las secuencias didácticas así diseñadas permiten disminuir el salto cognitivo necesario para adquirir ciertos conceptos, al proporcionar información adicional dentro de un contexto no matemático. Nuestra propuesta sienta por lo tanto una serie de fundamentos básicos y consideraciones para un diseño eficiente de secuencias didácticas haciendo uso de fragmentos de películas como recurso didáctico, subrayando tanto su finalidad motivadora como facilitadora de introducción de conceptos matemáticos.

Palabras clave: situaciones didácticas, transposición didáctica, cine, matemáticas.

SUMMARY: The use of cinema as didactical resource in the Mathematics classroom has interested several authors and teachers during the last years, mainly because of its power to motivate students. On this point, suggestive compilations of scenes containing mathematical references, detailed analysis of movies closely related to Mathematics and even didactical materials to be used in the classroom have been developed. This article proposes a theoretical framework for designing classroom sequences based on the didactical situation which can arise from movies or fiction series scenes. In order to develop such a framework, we follow a didactical engineering process, taking into account some specific characteristics, as the one related to the didactical transposition, as it is required to consider the mathematic knowledge within the chosen scene, overall the way it appears. As well, a classroom experience is described, designed following the mentioned guidelines and implemented in the course of a collaborative project between two secondary education centers, where a significant motivation increase has been detected, due to using mathematical situations from the real world (or from fictional contexts but which can be easily assimilated by the students). There was also evidence about the fact that the designed didactical sequences allow to reduce the cognitive gap required to acquire certain mathematical concepts, because of the scenes provide additional information within an extra-mathematical context. Therefore, our proposal establishes some basic considerations in order to efficiently design didactical sequences using movie scenes as a resource, underlining its power to motivate as well as its facilitating ability when introducing new mathematical concepts to our students.

Key words: didactical situations, didactical transposition, cinema, mathematics.

RÉSUMÉ: Divers auteurs et professeurs se sont intéressés dans les dernières années à l'usage du cinéma et les séries de TV comme ressource didactique aux Mathématiques dans l'éducation secondaire, il est dû principalement à la motivation que ce moyen produit chez les élèves. À ce sujet, des résumés suggestifs de scènes ont été élaborés avec des références mathématiques, des analyses détaillées de films avec une forte relation avec les mathématiques et, même, les matériels didactiques pour être employés dans la classe. L'article présent propose un cadre théorique initial pour le dessin de séquences de salle basées sur les situations didactiques qui peuvent être générées à partir des fragments de films ou de séries. Pour cela, on 
suit un processus d'ingénierie didactique, dans lequel certaines particularités sont tenues en compte, comme celle qui fait référence à la transposition didactique, car il devient nécessaire de considérer laquelle est la connaissance en jeu à l'intérieur du fragment et comment il apparaît. De la même manière, on présente une expérience de classe dessinée selon ce processus dans le sein d'un projet de collaboration entre deux centres d'éducation secondaire, où une augmentation a été vérifiée dans la motivation des élèves, grâce à travailler avec les situations mathématiques du monde réel (ou fictif mais assimilable par eux). Ils se sont aussi trouvés des indices de que les séquences didactiques telles dessinées permettent de diminuer le saut cognitif nécessaire pour acquérir des certains concepts, en fournissant de l'information additionnelle dans un contexte non mathématique. Notre proposition, par conséquent, vérifie une série de fondements basiques et de considérations pour un dessin efficient de séquences didactiques en utilisant des fragments de films comme ressource didactique, en soulignant tant son but motivant comme qui permet l'introduction de concepts mathématiques.

Mots clés: des situations didactiques, transposition didactique, cinéma, mathématiques.

\section{INTRODUCCIÓN}

Cada vez son más los profesores e investigadores interesados en el uso del cine como recurso didáctico en el aula de Matemáticas en educación secundaria. Todo docente que vea en ello un elemento motivador y una posibilidad de introducir conceptos matemáticos de modo diferente encontrará con facilidad colecciones de escenas con referencias matemáticas (Knill, 2013; Requena, 2013), páginas monotemáticas centradas en una serie en concreto (Cornell Department of Mathematics, 2010; Greenwald y Nestler, 2010) y libros que exploran a fondo esta simbiosis de cine y matemáticas (Población, 2006a; Polster y Ross, 2012). Por otra parte, existen varios autores y grupos de trabajo que, además de elaborar compilaciones de referencias, preparan actividades listas para su empleo en el aula o sugieren la aplicación didáctica de escenas y películas (Sorando, 2007; Raga, Muedra y Requena, 2009a y 2009b; Sorando, 2011, 2012; Martín y Martín, 2012; Roberts y Roberts, 2013).

Gómez Chacón (2005), analizando el informe PISA (2003) sobre la competencia matemática, subrayó la importancia de potenciar la motivación intrínseca en clase de Matemáticas. Para ello, sugirió una serie de estrategias, como enfatizar el valor de las matemáticas, fomentar el aprendizaje cooperativo, ayudar a los alumnos tanto a generar conocimiento matemático mediante su propia reflexión como a interiorizar metas de aprendizaje y trabajar el plano afectivo de los alumnos. El cine y las series constituyen uno de los medios de comunicación con más poder de seducción para nuestros alumnos y se postula como una herramienta muy interesante a incluir en el aula.

Desde nuestro punto de vista, se hace necesario el profundizar sobre un marco teórico que aborde la utilización didáctica del cine en Matemáticas, que analice cómo 
aprenden los alumnos y que permita el diseño de secuencias didácticas. A pesar de la relativa abundancia de referencias sobre compilaciones matemáticas y actividades para el aula, hemos encontrado pocos estudios en la bibliografía que se preocupen por este marco teórico o que analicen los procesos que tienen lugar en el aula. A este respecto, Palmer (1994) realiza una experiencia de aula, basada en la utilización de secuencias cortas de cine y expone en su trabajo las conclusiones obtenidas a partir de unos cuestionarios de opinión respondidos por los alumnos.

Coincidimos con la línea argumental de otros autores (Meyer, Dekker y Querelle, 2001), que recomiendan introducir los nuevos conceptos mediante situaciones de la vida real. Meyer et al., en su estudio, distinguen diferentes roles para el contexto en la educación matemática: para motivar, para aplicar, como fuente de conocimiento matemático, como fuente para hallar estrategias de resolución de problemas y como punto de anclaje para la comprensión del estudiante.

Se puede llevar a cabo una primera clasificación de las actividades típicas en función de su duración, ya que o bien se hace uso de escenas de corta duración o bien de películas completas. Tradicionalmente, debido a la amplitud del currículo, el visionado de películas completas se suele dejar para los días previos a vacaciones y se trabaja rápidamente sobre un cuestionario o un resumen. Los objetivos que se persiguen con la visualización de una película son diversos y atienden a los roles que desempeña el contexto que hemos comentado anteriormente. Dependiendo de la película escogida, el profesor puede pretender motivar a los alumnos, enseñarles situaciones reales de uso de las matemáticas, una visión histórica de las mismas, etc.

Estos mismos objetivos son abordables mediante la utilización de escenas o fragmentos de corta duración. El uso de fragmentos (no tiene por qué tratarse de una escena completa o restringirse a una única escena) permite tratar una mayor variedad de temas, optimizando además el tiempo empleado, por lo que en el presente artículo nos centraremos en la utilización de estos fragmentos. En cualquier caso, la metodología de diseño de actividades que propondremos se puede adaptar para el visionado de una película completa, aunque dado el tiempo de clase disponible es mucho más versátil el empleo de fragmentos.

Tal y como indica Población Saez (2006b), y es una opinión general, no basta con que el profesor entre en clase, proyecte una escena y deje que a partir de aquí entre en juego la improvisación. Si se improvisa, puede ocurrir cualquier cosa, como que la resolución de la actividad no sea tan trivial como parecía y que el profesor quede en evidencia, o como que la actividad no tenga nada que ver con lo que se está viendo en ese momento en clase. Para evitar situaciones como estas, en el presente artículo proponemos enmarcar teóricamente la utilización del cine como recurso didáctico dentro de la escuela francesa de la didáctica de las matemáticas, representada fundamentalmente por la teoría de las situaciones didácticas de Brousseau (1997) y la transposición didáctica de Chevallard (1985).

La labor de diseño irá encaminada, como veremos, a la creación de una secuencia didáctica, que permita incluir situaciones a-didácticas donde el alumno 
pueda elaborar su propio conocimiento interactuando con el denominado milieu o medio.

\section{BREVE ANÁLISIS DE LOS RECURSOS DISPONIBLES}

Antes de adentrarnos en el diseño de actividades, vamos a mostrar que existen suficientes y variadas referencias matemáticas en el cine como para que resulte de interés investigar su aplicación didáctica.

\subsection{Estudios sobre referencias matemáticas en el cine o las series}

La página Mathematical Fiction, que mantiene Alex Kasman (2012), incluye una compilación de más de 1.000 referencias matemáticas en diversos medios, incluyendo el cine y la televisión, categoría que aporta un centenar de entradas. La base de datos de dicha web es accesible bien mediante una búsqueda general o bien navegando por las diferentes categorías. Estas categorías incluyen una clasificación por el tipo de medio (online, cómics, novelas, películas, etc.), por género (aventura, educativo, humor, etc.), por el tema de la referencia matemática (álgebra, aritmética, probabilidad, etc.) o por el contexto (mujeres matemáticas, matemáticas antisociales, etc.). Desde 2012, Kasman ha comenzado a ofrecer además una serie de recomendaciones basada en el perfil del público. De esta forma, diferencia entre referencias que él recomendaría para niños, para adultos jóvenes, para estudiantes o profesores de matemáticas, para aficionados a la ciencia-ficción y para lectores.

En este sentido, el espacio web de Kasman (2012) (Figura 1) constituye la mayor base de datos disponible en Internet sobre referencias matemáticas en el cine, sólo comparable al trabajo de Población (2006a) o a la web de Polster y Ross (2012). No obstante, debemos tomar con cuidado estos datos, pues pueden estar sesgados por los gustos del autor o sus colaboradores. Baste observar que en la clasificación según el contexto (Figura 3) aparece una categoría para Sherlock Holmes. ¿Por qué para el famoso detective y no para Sheldon Cooper, de la serie The Big Bang Theory o para Charlie Eppes, de Numb3rs? Sin embargo, un análisis de la cantidad de entradas en términos absolutos (Figura 2) nos revela la abundancia de materiales con contenidos matemáticos para las principales ramas de las matemáticas, así como para las matemáticas aplicadas (informática, criptografía, física, etc.) y algunas subramas que en los últimos años han gozado de gran popularidad (caos, fractales, infinito). Si ahora nos fijamos en términos relativos, parece ser que la geometría, el álgebra, la aritmética y las matemáticas aplicadas sobresalen sobre los demás temas, por encima de las 100 entradas cada una. No obstante, debemos hacer una lectura cuidadosa, ya que por ejemplo el concepto del infinito aparece en una categoría propia, siendo que forma parte del cálculo diferencial. Sumando el número de entradas del infinito con las del cálculo diferencial obtendríamos una categoría sobresaliente, sobrepasando también las 100 entradas. 
Interfaz principal de la base de datos Mathematical Fiction (Kasman, 2012)

\begin{tabular}{l|l|l|l|l|l} 
Home & All & New & Browse & Search & About
\end{tabular}

\section{Browse the Mathematical Fiction}

\begin{tabular}{|c|c|}
\hline $\begin{array}{l}\text { Select a Medium } \\
\text { - Available Free Online (119 entries) } \\
\text { - Collection (12 entries) } \\
\text { - Comic Book (18 entries) } \\
\text { - Films (98 entries) } \\
\text { - Povels (501 entries) } \\
\text { - Plays (500k ( } 8 \text { entries) } \\
\text { - Short Stories (456 entries) } \\
\text { - Television Series or Episode (26 entries) }\end{array}$ & \begin{tabular}{||l||} 
Select a Genre \\
- Adventure/Espionage (78 entries) \\
- Children's Literature ( 82 entries) \\
- Didactic (79 entries) \\
- Fantasy (118 entries) \\
- Historical Fiction (152 entries) \\
- Horror ( 33 entries) \\
- Humorous ( 222 entries) \\
- Mystery (141 entries) \\
- Science Fiction (445 entries) \\
- Not Science-Fiction, Fantasy or Horror (579 entries)
\end{tabular} \\
\hline $\begin{array}{l}\text { Select a Motif } \\
\text { - Academia (196 entries) } \\
\text { - Aliens ( } 88 \text { entries) } \\
\text { - Anti-social Mathematicians (96 entries) } \\
\text { - Autism (15 entries) } \\
\text { - Cool/Heroic Mathematicians (41 entries) } \\
\text { - Evil mathematicians (44 entries) } \\
\text { - Female Mathematicians (167 entries) } \\
\text { - Future Prediction through Math (45 entries) } \\
\text { - Gödel ( } 37 \text { entries) } \\
\text { - Genius (49 entries) } \\
\text { - Higher/Lower Dimensions (69 entries) } \\
\text { - Insanity (70 entries) } \\
\text { - Math as Beautiful/Exciting/Useful (67 entries) } \\
\text { - Math as Cold/Dry/Useless ( } 32 \text { entries) } \\
\text { - Math Education (109 entries) } \\
\text { - Mobius Strip/Nonorientability ( } 31 \text { entries) } \\
\text { - Music (20 entries) } \\
\text { - Newton (15 entries) } \\
\text { - Prodigies ( } 66 \text { entries) } \\
\text { - Rroving Theorems (97 entries) } \\
\text { - Real Mathematician (110 entries) } \\
\text { - Romance (190 entries) } \\
\text { - Sherlock Holmes (14 entries) } \\
\text { - Time Travel (52 entries) } \\
\text { - Turing (23 entries) } \\
\text { - War (47 entries) }\end{array}$ & \begin{tabular}{||l} 
Select a Topic \\
- Algebra/Arithmetic/Number Theory (178 entries) \\
- Analysis/Calculus/Differential ( 83 entries) \\
- Chaos/Fractals (61 entries) \\
- Computers/Cryptography (102 entries) \\
- Geomal Mathematics ( 46 entries) \\
- Infinity ( 49 entries) \\
- Logic/Set Theory ( 94 entries) \\
- Mathematical Finance ( 28 entries) \\
- Mathematical Physics (142 entries) \\
- Rrobability/Statistics (66 entries) \\
Real Mathematics (134 entries) \\
\end{tabular} \\
\hline \multicolumn{2}{|c|}{$\begin{array}{r}\text { View the Whole Database By: } \\
\text { Title or Author or Year }\end{array}$} \\
\hline
\end{tabular}


FIGURA 2

Número de referencias matemáticas clasificadas por ramas o conceptos de las matemáticas en el sitio web de Kasman (2012)

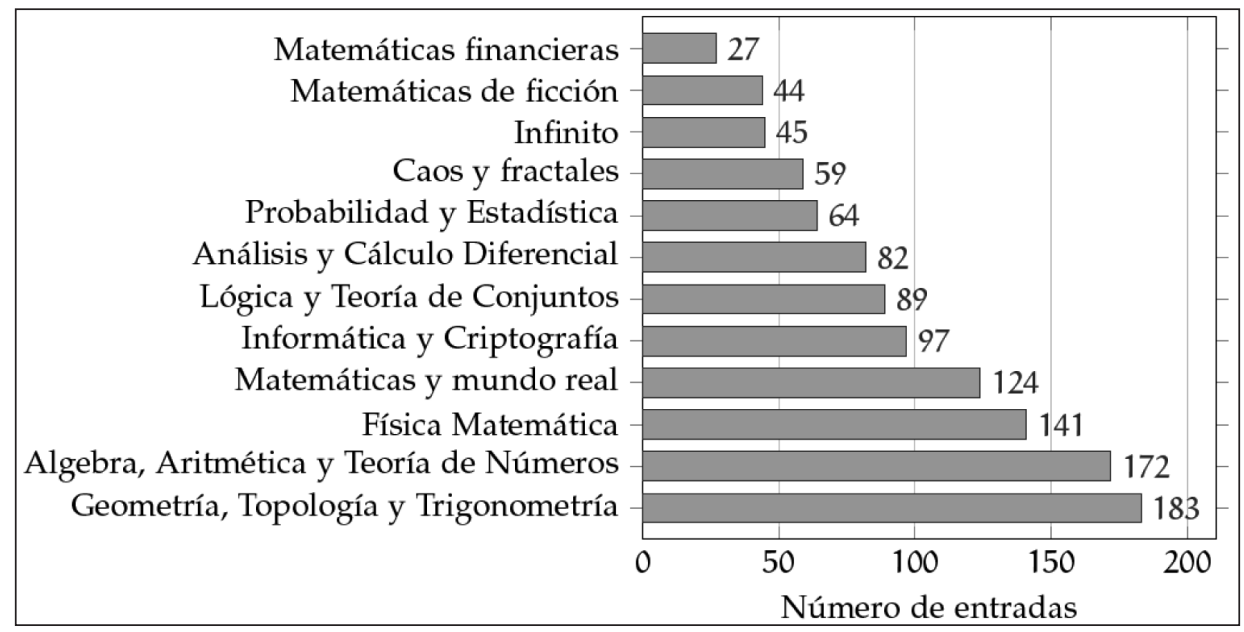

En cuanto al contexto en el que se desarrollan los materiales compilados por Kasman (Figura 3), las categorías más prominentes son el mundo académico, romance y mujeres matemáticas, cada una con más de 150 entradas. Les siguen de cerca, superando las 100 entradas, los personajes matemáticos reales, la religión y la educación matemática. Resulta de interés para nuestro trabajo el observar la cantidad de contextos diferentes en los que se pueden encontrar referencias matemáticas, sobre todo, si al usar o recomendar estos materiales en clase, el profesor desea tratar un tema transversal en concreto.

Un aspecto que distingue a este sitio web es que, además de incluir un resumen y una pequeña explicación para cada referencia, incluye un sistema de votos con el fin de puntuar de forma separada el contenido matemático de la calidad cultural (literary quality). Además, proporciona una lista de trabajos similares con los que seguir explorando la base de datos.

Otro referente es el espacio web australiano The Mathematical Movie Database (Polster y Ross, 2012), que ofrece una compilación excelente de referencias matemáticas, aunque carece de la agilidad de búsqueda de la web de Kasman. Ahora bien, este hecho queda compensado con creces al incluir más de 800 referencias centradas exclusivamente en el cine y las series de televisión, lo que constituye un excelente recurso. 


\section{FIGURA 3}

Número de referencias matemáticas clasificadas por temática argumental de la película o serie en el sitio web de Kasman (2012)

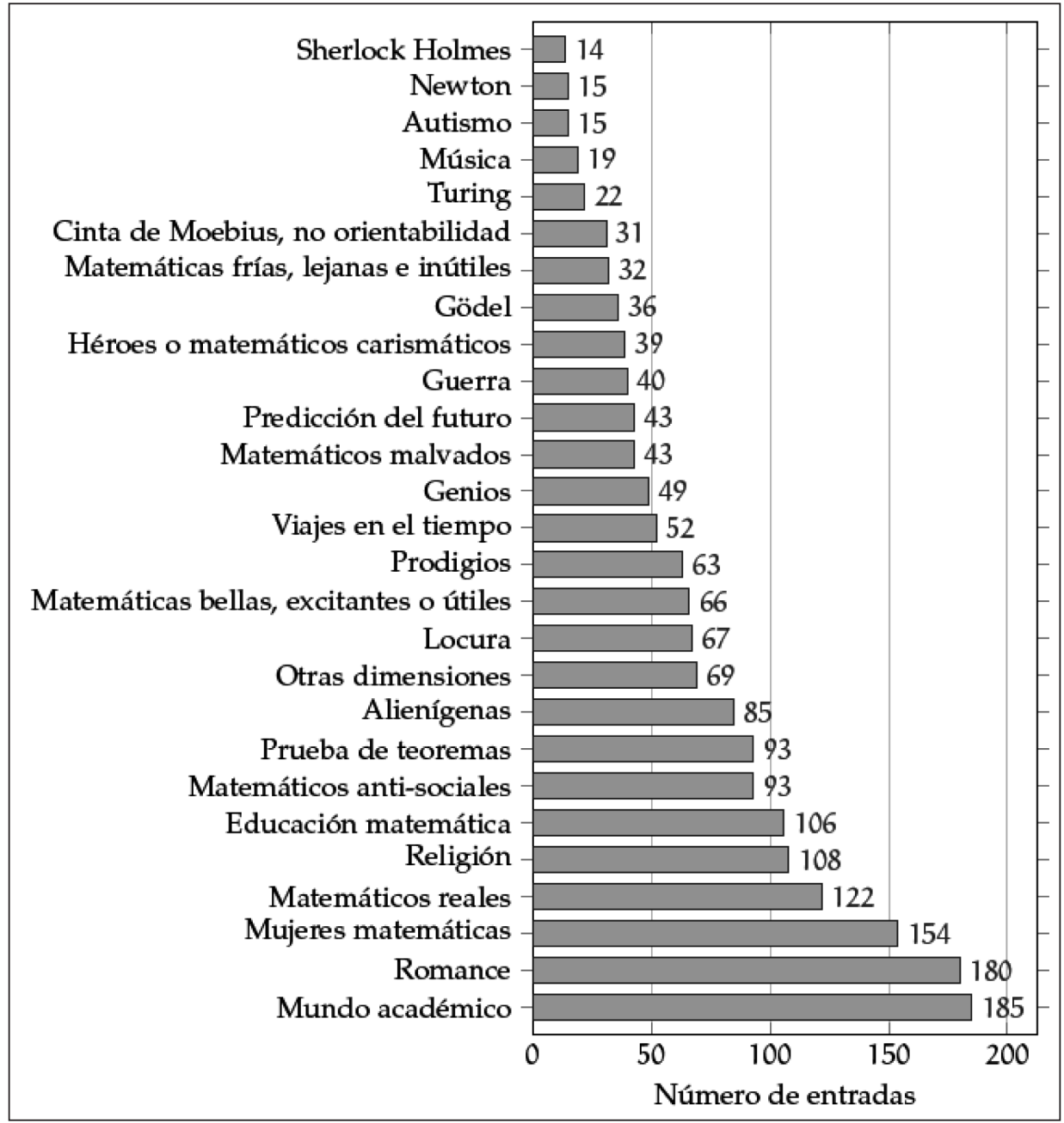

\subsection{Clasificación de los fragmentos}

Podría efectuarse una clasificación general de fragmentos de películas o series de televisión con referencias matemáticas aptas para su uso en el aula, en función del tipo de cuestión, problema o secuencia didáctica que puedan generar. Se hace 
necesario llevar a cabo esta clasificación, ya que, en función del tipo de referencia matemática, el diseño de la ingeniería didáctica dará más peso específico a unas situaciones frente a otras. En las compilaciones y repositorios de recursos didácticos nos encontramos con los siguientes tipos fundamentales de escenas, remitiendo para una mayor profundización a Sorando (2007 y 2012):

FIGURA 4

Clasificación de los fragmentos extraídos de películas y series con referencias matemáticas, en función del tipo de uso didáctico que puede hacerse

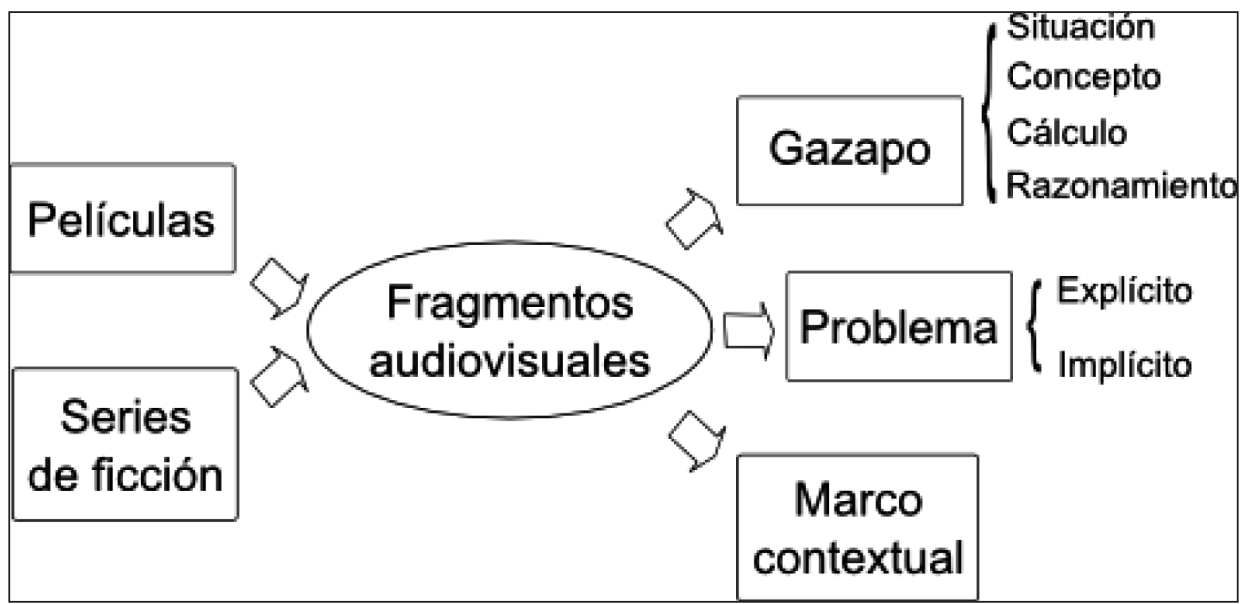

\subsubsection{Errores o gazapos}

En esta clase de escenas se produce un error matemático. Suele aparecer en los diálogos de algún personaje, bien de forma involuntaria por error del actor o del equipo de producción; bien de forma voluntaria, como exigencia del guión. No obstante, el error también puede encontrarse en un objeto que aparezca en escena, una pizarra que forme parte del escenario, etc. Esta distinción se corresponde con la que ya hiciera Sorando (2007), que diferencia entre gazapos de situación y gazapos de concepto, de cálculo y de razonamiento.

Un ejemplo de gazapo de concepto lo encontramos en la escena del reparto del botín en Granujas de medio pelo (Woody Allen, 2000), en la que un personaje comete varios errores acerca de las fracciones por exigencias del guión. Un error de cálculo totalmente involuntario aparece en la película 21 Blackjack (Robert Luketic, 2008), donde el protagonista, dependiente de una tienda de ropa, calcula la cuenta mentalmente, incluyendo descuentos variados. Esta última escena ofrece una oportunidad excelente en el marco de un proyecto colaborativo a través de 
PABLO BELTRÁN PELLICER Y ANNA ASTI

UTILIZACIÓN DIDÁCTICA DEL CINE EN MATEMÁTICAS

Internet con estudiantes de diferentes nacionalidades, pues las versiones dobladas cambian el resultado final.

La actividad natural que surge de este escenario se suele denominar caza del gazapo, y consiste en la búsqueda de dichos errores. Si para descubrir el gazapo hay que resolver un problema, tendríamos un híbrido entre este tipo de escena y el siguiente.

\subsubsection{Resolución de problemas}

Hay escenas en las que se plantea explícitamente un problema matemático en toda regla. La serie de televisión Numb3rs es una fuente inagotable (Devlin y Lorden, 2007), aunque no la única, de este tipo de escenas. Prácticamente todos sus capítulos reducen la investigación de un crimen a un problema matemático. No obstante, hay muchas otras fuentes de este tipo de escenas. Es representativo el siguiente ejemplo, tomado de la serie de dibujos animados Futurama (Matt Groening, 1999) y que constituye ya un clásico recurso en el aula para los iniciados en el uso del cine como recurso didáctico en Matemáticas. El personaje llamado Fry resulta congelado accidentalmente por un período de 1.000 años. Al cabo de ese tiempo acude al banco donde tenía su cuenta corriente. Cuál será su sorpresa cuando la cajera le anuncia que el saldo que tenía de 93 centavos, con un interés anual del 2,25\%, hace un total de 4.300 millones de dólares. En este caso la actividad que se suele proponer a los alumnos es comprobar si la cuenta está bien hecha y probar con otros períodos de tiempo y otros intereses (Raga, Muedra y Requena, 2009a y 2009b; Sorando, 2011).

Otras escenas, en cambio, pueden esconder problemas matemáticos de forma implícita y se hace necesario, no ya sólo recomendable, que el profesor exponga el problema a resolver.

\subsubsection{El fragmento como marco contextual}

En ocasiones, el único uso que se puede dar a un fragmento consiste en mostrar la relación con el mundo real y, a partir de ahí, plantear un problema centrado en el tema. Nótese que no es lo mismo que el caso de los problemas implícitos, ya que aquí no es el fragmento en sí el que genera el problema. Por ejemplo, si queremos proponer un problema sobre intereses compuestos, podemos poner antes una pequeña escena de Wall Street (Oliver Stone, 1987) o de alguna otra película donde aparezcan escenas de bancos o de la bolsa.

\section{PROPUESTA DE UN MARCO TEÓRICO PARA EL DISEÑO DE SECUENCIAS DidÁCTICAS}

Es un hecho reconocido que los medios audiovisuales en general constituyen tanto un objeto de estudio como un instrumento, un recurso de enseñanzaaprendizaje como el que analizamos en este artículo. Sin embargo, como señalan Spanhel (2011) y Ballesta y Guardiola (2001) su utilización en el aula está lejos 
todavía de ser una práctica generalizada, bien por falta de formación al respecto, el no disponer de la infraestructura adecuada o porque ello exigiría un cambio drástico en sus modelos de enseñanza.

Los fragmentos de películas y series forman un subconjunto con entidad propia dentro de los medios audiovisuales. El lenguaje audiovisual posee unas características que lo hacen especial y que estimula la motivación. No abundan las descripciones rigurosas de experiencias que integren los medios audiovisuales en el aula de Matemáticas, y menos aún las que se refieran explícitamente al empleo del cine o las series. Por ejemplo, el trabajo de Bartolomé y Mateo (1983) analiza el uso de montajes audiovisuales creados específicamente para este fin (en concreto, para la enseñanza de la Estadística), mostrando que éstos incidían positivamente en la motivación de los estudiantes, a la vez que se intuía una tendencia también positiva, pero no concluyente, en términos de mejora del rendimiento. En la misma línea es el estudio de Palmer (1994), que utiliza pequeños fragmentos grabados a propósito para ilustrar las matemáticas dentro de un contexto determinado en el mundo real. De Pablos (1989) señala las diferencias entre el cine didáctico y el cine de ficción, desde el punto de vista diegético y subraya que tiene como objetivo final facilitar la elaboración de conocimiento por parte de los alumnos, más que mostrar un conocimiento. Nuestra propuesta asimila dicho objetivo al diseño de secuencias de clase basadas en fragmentos extraídos de películas y series de ficción. Además, como hemos comentado, actualmente se dispone de recursos que posibilitan de una manera sencilla el empleo de fragmentos extraídos de películas y series; es decir, no creados ex profeso y que son más cercanos y estimulantes para nuestro alumnado. No obstante, los dos tipos de fragmentos no son en absoluto excluyentes, pudiendo complementarse en la práctica.

\subsection{Transposición didáctica}

Chevallard (1985) toma el concepto de transposición didáctica de Verret (1973) y lo adapta a la didáctica de las matemáticas, desarrollando la perspectiva antropológica de las tesis originales de Verret. Para Chevallard (1985), es fundamental el estudio de lo que él denomina el sistema didáctico, formado por el profesor, los alumnos y un saber matemático determinado. Ahora bien, hay que distinguir entre el saber matemático erudito, como el que se puede estudiar en etapas universitarias, aceptado en el mundo académico, y el saber matemático a enseñar. Es el proceso de adaptación entre las dos formas de saber lo que se denomina transposición didáctica y es labor del profesor el realizar dicho proceso convenientemente, teniendo en cuenta las características de sus alumnos.

Cuando un profesor decide emplear una escena como recurso didáctico ha de realizar un ejercicio de transposición, condicionado a dicha escena en particular. Esto es así porque, aunque la escena en cuestión no puede modificarse, sí debe reflexionarse acerca del modo de presentarla a los alumnos y sobre cómo trabajar con ella. El profesor, al ver la escena, identificará el saber matemático involucrado. Dicho saber se hallará, con toda seguridad, en un punto intermedio entre el 
saber erudito y el saber enseñar, que podemos representar mediante el siguiente diagrama (Figura 5):

\section{FIGURA 5}

Proceso de transposición didáctica en el diseño de secuencias de aula haciendo uso de fragmentos de películas

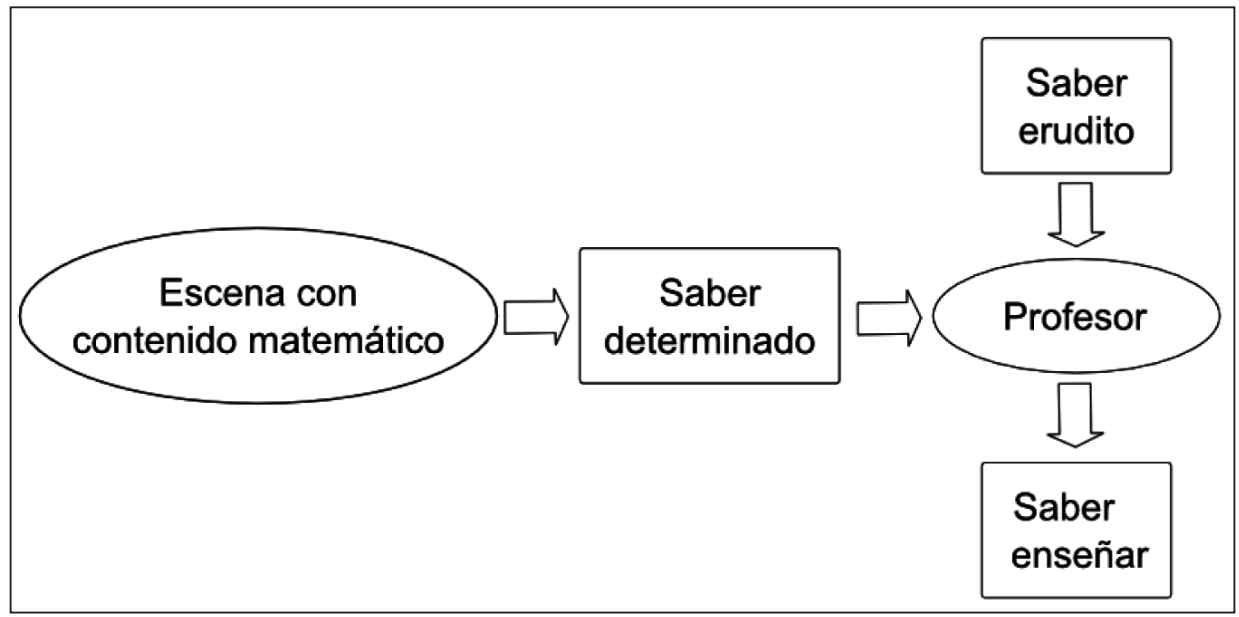

\subsection{Situaciones didácticas}

Para Brousseau (1997), el estudio de cualquier situación de enseñanza-aprendizaje implica el análisis del sistema formado por el profesor, los alumnos y el milieu o medio. El milieu es el entorno; esto es, cualquier elemento que interactúa con el alumno. Se trata de un concepto fundamental en la teoría de las situaciones didácticas, ya que el estudio de las situaciones puede reducirse prácticamente al estudio de las relaciones del alumno con el milieu.

El hecho de introducir un fragmento de una película o serie como elemento del milieu añade un punto de anclaje donde el alumno pueda construir el conocimiento. Bien sea porque la escena ilustra de forma visual una aplicación práctica de las matemáticas o porque el alumno se identifique con alguno de los personajes, la utilización de este recurso despierta, cuando menos, la curiosidad del alumno.

El trabajo de Billet (1996) profundiza en cómo se produce la construcción del conocimiento en contextos socioculturales, y propone un modelo que considera el aprendizaje como una apropiación personal de formas sociales de ese mismo conocimiento.

Dicho proceso de apropiación implica una transformación del conocimiento social, que implica que el conocimiento personal se construye a partir de la interacción 
de dicho conocimiento social con los sistemas simbólicos y de interpretación de uno mismo. Por ejemplo, la resolución de problemas de forma colaborativa permite la adquisición de conocimiento interactuando con los compañeros y con el medio que introduce el problema a resolver, así como con el contexto en el que se presenta. Las ideas de Billet referentes al empleo del contexto enlazan con los fundamentos de la Educación Matemática Realista, cuyo origen son los trabajos de Freudenthal (1991).

En resumen, la construcción de conocimiento al usar películas o series como recurso didáctico es posible gracias a la integración de la propia realidad del alumno con el escenario presentado a través de la pantalla. De esta manera, se promueve cierta sensibilidad hacia el objeto de conocimiento, a la vez que se facilita su percepción.

\section{EXPERIENCIA EN EL MARCO DE UN PROYECTO ETwINNING COLABORATIVO}

\subsection{Participantes}

La experiencia que vamos a describir se llevó a cabo durante la segunda mitad del curso 2011-2012 en el marco del proyecto colaborativo eTwinning 7art Maths, entre un centro educativo español y otro italiano (Asti y Beltrán, 2012), con alumnos de primer curso de ESO. Dicho proyecto recibió los sellos de calidad del programa, tanto los nacionales como el europeo, así como el premio nacional de eTwinning Italia.

Participaron en total 49 alumnos divididos en tres grupos de la siguiente forma: un grupo de 21 alumnos en el centro italiano y dos grupos de 14 alumnos cada uno en el español. La edad del 98,4\% de los alumnos era de 12-13 años, salvo el $1,6 \%$ restante que eran repetidores, con una edad de 13-14 años.

\subsection{Descripción del proyecto}

El proyecto fue diseñado por los autores, quienes se pusieron en contacto a través de los propios foros de la plataforma virtual eTwinning. Además, colaboraron los profesores del departamento de Inglés y diversos profesores de apoyo. Mediante la contextualización de ciertas actividades en clase de Matemáticas empleando fragmentos de películas y series, nos planteamos los siguientes objetivos:

1. Incrementar la motivación de los alumnos en Matemáticas.

2. Facilitar el aprendizaje de conceptos matemáticos, contextualizando la enseñanza de las matemáticas y presentando aplicaciones en el mundo cercano al alumno.

3. Alineación de las secuencias didácticas con el currículo.

4. Impulsar el empleo de una lengua extranjera (inglés) para comunicarse con otros estudiantes europeos. 
La gestión se realizó de forma colaborativa haciendo uso intensivo de las herramientas online de Google Drive. En el documento de proyecto se especificaron los tipos de actividades a trabajar, así como los puntos curriculares coincidentes en todos los grupos participantes. De esta manera, se dividió el proyecto en cuatro bloques diferenciados:

\section{TABLA 1}

División del proyecto en bloques temáticos,

indicando los recursos virtuales empleados y las actividades presenciales

\begin{tabular}{|c|c|c|c|}
\hline BLOQUE & TAREA & $\begin{array}{l}\text { RECURSOS } \\
\text { VIRTUALES }\end{array}$ & ACTIVIDADES PRESENCIALES \\
\hline Inicial & $\begin{array}{l}\text { Realización de un póster } \\
\text { colaborativo en red, con } \\
\text { el fin de conocer a los } \\
\text { demás participantes. }\end{array}$ & $\begin{array}{l}\text { Herramientas } \\
\text { ofimáticas de } \\
\text { Google Drive, } \\
\text { chat y foros }\end{array}$ & Exposición del póster. \\
\hline Escenas & $\begin{array}{l}\text { Resolución de } \\
\text { problemas matemáticos } \\
\text { basados en las escenas. }\end{array}$ & Chat y foros & $\begin{array}{l}\text { Visualización de las escenas. } \\
\text { Puesta en común de los } \\
\text { resultados de cada grupo. } \\
\text { Institucionalización por parte } \\
\text { del profesor. }\end{array}$ \\
\hline Actores & $\begin{array}{l}\text { Investigación de } \\
\text { la relación con las } \\
\text { matemáticas de ciertos } \\
\text { actores, directores y } \\
\text { guionistas. }\end{array}$ & $\begin{array}{l}\text { Herramientas } \\
\text { ofimáticas de } \\
\text { Google Drive, } \\
\text { chat y foros }\end{array}$ & $\begin{array}{l}\text { Exposición de las } \\
\text { presentaciones por parte de } \\
\text { los alumnos. }\end{array}$ \\
\hline Película & $\begin{array}{l}\text { Resolución de } \\
\text { problemas basados en la } \\
\text { película. }\end{array}$ & Chat y foros & $\begin{array}{l}\text { Proyección de la película. } \\
\text { Puesta en común de los } \\
\text { resultados de cada grupo. } \\
\text { Institucionalización por parte } \\
\text { del profesor. }\end{array}$ \\
\hline Final & $\begin{array}{l}\text { Evaluación del proyecto } \\
\text { mediante cuestionario } \\
\text { abierto y despedida. }\end{array}$ & Chat y foros & $\begin{array}{l}\text { Realización del cuestionario de } \\
\text { evaluación. } \\
\text { Entrevistas a alumnos } \\
\text { seleccionados. }\end{array}$ \\
\hline
\end{tabular}

El objetivo de las actividades del bloque inicial consistió en formar los grupos de trabajo, tratándose contenidos puramente transversales, como el uso del inglés dentro de un contexto virtual. El bloque de escenas es el de mayor interés para nuestro artículo, ya que es el que engloba las actividades basadas en fragmentos de películas y series. En las actividades del bloque de actores se vuelven a tratar contenidos transversales, relacionando las matemáticas con profesionales del cine y la televisión, humanizándolas de alguna manera para el estudiantado. Los alumnos tuvieron que investigar en Internet la biografía de actores, directores o guionistas, 
para terminar realizando una presentación en clase. El bloque de película es similar al dedicado a escenas, con la diferencia de que se proyectó una película completa, Cielo de octubre (Joe Johnston, 1999), con gran contenido matemático y motivacional, a modo de colofón del proyecto. Tras su proyección, se inició un debate guiado en los foros virtuales y se plantearon una serie de actividades similares al del bloque de escenas. Por último, el bloque final consistió en la evaluación del proyecto, e incluyó la realización de un cuestionario y una serie de entrevistas a ciertos alumnos.

En lo sucesivo nos centraremos en la descripción del diseño e implementación de las actividades del bloque de escenas, que siguieron el mismo patrón de trabajo en todas ellas. Los tres grupos veían los fragmentos audiovisuales en el aula, durante el tiempo de clase ordinario y, a continuación, se les presentaba la actividad a desarrollar en su idioma nativo. La secuencia didáctica continuaba por la tarde o durante el fin de semana de forma online, para finalizar de nuevo en el aula al día siguiente. Empleando terminología propia de la teoría de las situaciones didácticas, la situación inicial de acción y la institucionalización final tenían lugar en el aula física, mientras que las situaciones de comunicación y validación se realizaban tanto online en idioma inglés como de forma presencial (Figura 6).

\section{FIGURA 6}

Descripción del patrón de trabajo de las actividades en términos de la Teoría de las Situaciones Didácticas

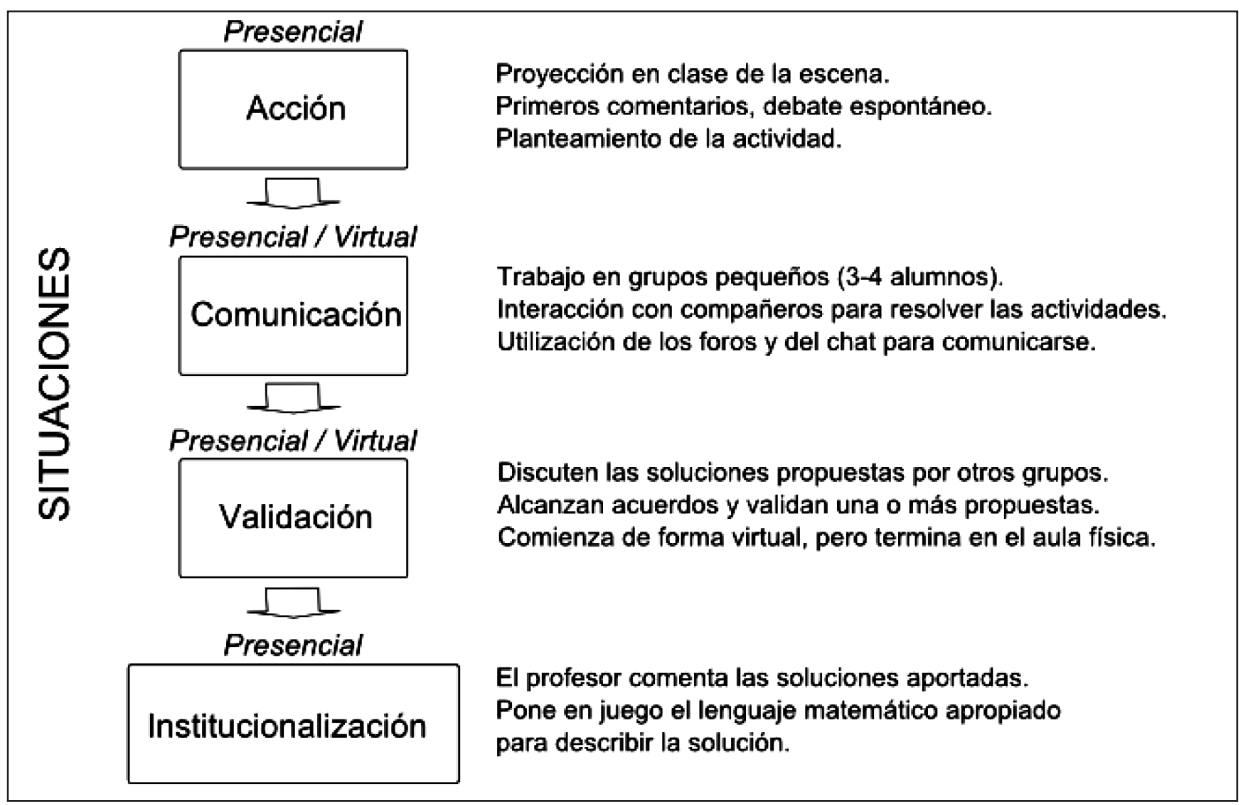




\subsection{Instrumentos de investigación}

La metodología de investigación empleada es de carácter cualitativo descriptivo. Todas las actividades realizadas por los alumnos en el espacio virtual de eTwinning quedan recogidas en sus producciones: discusiones en el foro, mensajes en los chats y documentos ofimáticos de Google Drive. Por otro lado, los profesores anotaron el cumplimiento de diversos indicadores para constatar el efecto del proyecto en el estudiantado a nivel motivacional, de empleo de nuevas tecnologías y de rendimiento académico. Dichos indicadores son los recomendados en las especificaciones MICE-T (Model Instruments for a Common Evaluation, 2000), nacidas en el seno de una iniciativa Socrates Comenius para la autoevaluación de proyectos educativos. Se consideró el impacto en los alumnos, en los profesores y en la comunidad educativa en general, así como la gestión del proyecto.

Finalmente, se aplicó un cuestionario abierto a los participantes (basado también en MICE-T) y entrevistas semiestructuradas a estudiantes seleccionados, con el objetivo de evaluar la percepción del proyecto por el estudiantado. Dicho cuestionario constituía la última actividad del proyecto, por lo que se realizó en clase en presencia de los profesores, los cuales incidieron en que se contestasen todas las preguntas.

\subsection{Desarrollo del proyecto}

A continuación, describimos dos de las secuencias didácticas representativas del bloque de escenas:

\subsubsection{Jungla de cristal III: resolución de problemas}

En la escena seleccionada, los protagonistas tienen que resolver un problema de lógica para evitar que explote una bomba en un parque. Es un buen ejemplo de la aparición de las matemáticas de forma explícita dentro del cine, y aparece referenciada en la práctica totalidad de las fuentes mencionadas anteriormente. Además, el acertijo en sí se incluye en multitud de libros de texto de secundaria y, por otro lado, es una escena atractiva para nuestros alumnos, ya que aparece en una película de acción de gran impacto comercial.

El problema consiste en conseguir 4 galones exactos de agua, permitiéndose únicamente el empleo de una garrafa de 3 galones y otra de 5 galones. Hay varias formas de resolverlo, bien sea por ensayo y error o mediante ecuaciones diofánticas. Dado el nivel educativo y que prácticamente no se ha tratado el lenguaje algebraico todavía, el problema posibilita trabajar el pensamiento lógico, técnicas de resolución de problemas y, sobre todo, la actitud a la hora de afrontarlos. A continuación, detallamos la secuencia didáctica implementada:

Después de ver la escena en el aula, al final de la sesión, se dio un pequeño margen de tiempo para que los alumnos comentaran la solución. En la propia 
escena, los protagonistas solucionan el problema, pero el procedimiento que emplean no queda claro del todo:

— ¡Ya está! Aquí hay 2 galones, ¿no?

-Dejando un galón de espacio vacío.

-Aquí hay 5 galones. Si echamos un galón allá...

— ¡Nos quedan 4 galones justos!

—iŔpido!

—No lo derrames!

Es decir, los protagonistas no explican cómo consiguen llegar a tener los dos galones exactos, así que nuestros alumnos lo primero que piden es volver a ver la escena. Sin embargo, se les propone que la visualicen de nuevo en casa, ya que está disponible en el foro del proyecto, y que ahora trabajen en pequeños grupos la solución durante no más de los 10 minutos restantes de clase. Hasta aquí, los alumnos se han posicionado en las situaciones de acción y de comunicación. La situación de comunicación prosigue con la explicación de la solución personal de cada uno en la plataforma virtual y enlaza con la de validación, ya que los propios alumnos se dan cuenta de si una solución es válida o no, y así lo indican en sus respuestas o en clase al día siguiente, que es donde termina la validación y donde tiene lugar la institucionalización por parte del profesor.

Dos alumnos repiten la solución inicial -errónea- de los protagonistas:

Llena la garrafa pequeña y echa los 3 galones a la de 5 , entonces rellena una tercera parte de la de 3 galones y añádelo a la de 5. Espero que sea así...

La mayoría de los alumnos que respondieron en el foro (12) se aprovechan del estado intermedio del problema que muestra la escena, sin indicar cómo aparecen los dos litros en la garrafa de tres:

Como ellos dicen, en la botella de 3 litros hay 2 litros, así que no tienes más que poner un litro de la botella de 5 en la de 3 , y así te quedarán 4 litros en la de 5 .

Por lo tanto, se hace necesaria la intervención del profesor para indicar que se trata de una solución incompleta. Se les anima añadiendo el dato de que realmente hay infinitas soluciones.

Dos alumnos aportan finalmente la solución correcta. Es decir, los dos galones (o litros, según doblaje o interpretación de los alumnos) vienen de llenar la garrafa grande y vaciarla en la pequeña, con lo que quedan dos galones exactos, que luego se pueden echar a la pequeña.

En la institucionalización, al día siguiente en clase, se comenta la solución de la película y se explicita la ecuación diofántica $3 x+5 y=4$. Aunque realmente se trata de un contenido no incluido en el currículo, nuestra intención es únicamente utilizar la ecuación para mostrar el potencial del lenguaje algebraico.

La semana siguiente, y para trabajar la transversalidad de contenidos con la asignatura de inglés, el grupo español trabajó con una parodia de la escena del 
dúo de humoristas Cruz y Raya, en la que el problema consiste en conseguir 8 litros con una garrafa de 5 y otra de 3. Los alumnos subtitularon fragmentos del vídeo de forma colaborativa mediante un documento compartido en Google Drive. Posteriormente, un alumno voluntario, con ayuda del profesor, creó el vídeo subtitulado en el servicio gratuito online de Universal Subtitles. De esta forma, pudieron ver la parodia sus compañeros italianos. La famosa coletilla de la escena original, "Piensa McClane, piensa", se convirtió en un recordatorio espontáneo de la actitud a tomar frente a los problemas matemáticos: perseverar y buscar soluciones alternativas.

\subsubsection{Granujas de medio pelo: reparto de un botín}

Se trata de un claro ejemplo de búsqueda del error, en este caso, deliberado. Cuatro aspirantes a ladrones elucubran sobre cómo repartirse el botín una vez hayan perpetrado el atraco. Sin embargo, cuando uno de ellos propone incluir a su mujer en el reparto, pues hace de tapadera, las cuentas parecen complicarse y uno de ellos dice:

-Que la chica cobre una parte, pero no una parte entera.

- ¿Qué tal si todos cobramos un cuarto y ella, digamos, un tercio?

— ¡Tú estás "chinao"! Entonces cobraría más que nosotros.

- ¿Cómo lo sabes?

—Además, ¿¿de dónde sacas cuatro cuartos y un tercio? ¿No sabes sumar?

-Mira, yo en quebrados no me meto, ¿vale?

Para entender la escena y descubrir el error que comete el ladrón, es necesario conocer ciertos aspectos sobre las fracciones. Por un lado, qué son las fracciones, cómo leerlas y cómo se comparan y, por otro, cómo dividir un total en partes iguales. Finalmente, para explicar correctamente el gazapo es necesario saber sumar fracciones e, idealmente, llegar al concepto de las fracciones impropias, donde el numerador es mayor que el denominador.

Comúnmente, en los libros de texto de Matemáticas se comienza presentando las fracciones como partes de un todo. De esta forma, se plantean al alumno diferentes representaciones gráficas que, generalmente, son círculos o rectángulos. De ahí que el primer obstáculo serio que aparezca sea precisamente el concepto de fracción impropia, noción que permitirá a la vez una primera clasificación de las fracciones.

En esta ocasión, casi todos los alumnos descubrieron el error y es notable, a nuestro parecer, que la mayoría de ellos hayan optado por una respuesta que implique un método de comparar fracciones, sin necesidad de realizar la división. Sin embargo, las formulaciones de la explicación son, en ocasiones, incorrectas o incompletas. Por ejemplo, se puede adivinar que el alumno entiende de alguna manera el error, pero no es capaz de expresarlo: 
El error es que 4/4 no se puede comparar con 1/3, la operación es imposible de hacer.

Hay alumnos que utilizan sus conocimientos de números decimales, lo que da lugar a una explicación correcta, pero no satisfactoria, ya que se busca la explicación por medio de los fundamentos de las fracciones:

El ladrón cree que un tercio es más pequeño que un cuarto, pero no se da cuenta de que Frenchy conseguiría más dinero. De hecho: un tercio de 2M es 666.666,66 y un cuarto de $2 \mathrm{M}$ es 500.000 .

O más ingeniosas, pero en la línea de utilizar procedimientos que no implican fracciones:

Hay que dividir 2.000.000 entre 4, que da 500.000. Entonces de cada 500.000 quitamos 100.000, lo que da 400.000 para Frenchy, igual que a todos.

Y también hay alguna respuesta incorrecta, que demuestra que ese alumno no acaba de entender el final de la conversación de los ladrones:

El error lo comete el chico porque si le dan un tercio a Frenchy y ellos tienen un cuarto, ellos reciben menos porque un tercio es mayor que un cuarto.

Como hemos visto, este breve fragmento da pie al tratamiento de las fracciones impropias a partir de un simple reparto. El contexto de la escena hace que los gazapos que comente el personaje sean evidentes, lo que proporciona una información adicional del objeto matemático en juego y, de esta manera, disminuye el salto cognitivo necesario para la apropiación del conocimiento en concreto.

\subsection{Evaluación final y resultados}

Como el cuestionario final era semiestructurado y había varias cuestiones abiertas, hemos recogido las opiniones de los alumnos en una serie de categorías. A continuación, mostramos dicha información enriquecida con la obtenida de las entrevistas y el registro de observaciones de los profesores:

a) El proyecto se extendió a lo largo de unas 10 horas de clase en total, extendidas en sesiones de unos 20 minutos. La percepción del tiempo de clase dedicado al proyecto por el estudiantado osciló entre las 8 y las 12 horas, con una media de 9,6 horas. Aquellos alumnos que mostraron un mayor interés escribieron menos horas, ya que realmente querrían haber dedicado más tiempo.

b) Las actividades estaban perfectamente alineadas con los contenidos oficiales de los bloques de números y de álgebra y los alumnos así lo entendieron. 
TABLA 2

Contenidos de la asignatura tratados a lo largo del proyecto, según la percepción del estudiantado

\begin{tabular}{|l|l|l|l|}
\hline \multicolumn{1}{|c|}{ NÚMEROS } & \multicolumn{1}{|c|}{ ÁLGEBRA } & \multicolumn{1}{c|}{ COMUNES } & \multicolumn{1}{c|}{ TRANSVERSALES } \\
\hline $\begin{array}{l}\text { Operaciones } \\
\text { Fracciones y } \\
\text { operaciones } \\
\text { Porcentajes } \\
\text { Cálculos mentales }\end{array}$ & $\begin{array}{l}\text { Problemas con lógica } \\
\text { Ecuaciones }\end{array}$ & $\begin{array}{l}\text { Ejemplos en los vídeos } \\
\text { Aplicaciones de las } \\
\text { Matemáticas }\end{array}$ & \\
\hline
\end{tabular}

c) Todos los alumnos coincidieron en afirmar que a lo largo de las actividades se mostraron aplicaciones de las matemáticas en la vida normal o real, por lo que el proyecto ofreció una mejor perspectiva de las aplicaciones prácticas de la asignatura.

d) Prácticamente todos los alumnos indicaron que el proyecto les gustó, por diferentes motivos (Tabla 3).

TABLA 3

Valoración del grado de satisfacción del proyecto por los alumnos

\begin{tabular}{|l|c|}
\hline \multicolumn{1}{|c|}{ Sí } & \multicolumn{1}{|c|}{ No } \\
\hline \multicolumn{1}{|c|}{$90 \%$} & $10 \%$ \\
\hline Ha sido divertido & No me gusta participar \\
Hemos aprendido & \\
Ha sido útil & \\
Es fácil \\
Hemos usado el ordenador
\end{tabular}

e) La percepción acerca de la propia participación de los alumnos en el proyecto coincidió con las observaciones de los profesores. Ésta fue sensiblemente mayor que otras actividades de Matemáticas o de otras asignaturas. No obstante, un 20,4\% de los alumnos indicó que sólo participó al principio y un $12,2 \%$ que no participaron.

TABLA 4

Valoración del grado de participación del proyecto por los alumnos

\begin{tabular}{|c|c|c|}
\hline Sí & SÓLO AL COMIENZO & NO \\
\hline $67,4 \%$ & $20,4 \%$ & $12,2 \%$ \\
\hline
\end{tabular}

f) El $65,4 \%$ de los alumnos indicó que mejoraría el proyecto de alguna manera. Es interesante que la sugerencia que más se repitió fue la de que puntuase más el proyecto para la calificación de la asignatura. 
TABLA 5

Necesidad de mejora del proyecto y sugerencias por los alumnos

\begin{tabular}{|l|c|c|}
\hline \multicolumn{1}{|c|}{ Sí } & NO & NS/NC \\
\hline \multicolumn{1}{|c|}{$65,4 \%$} & $10,2 \%$ & $24,4 \%$ \\
\hline $\begin{array}{l}\text { Que contase más la nota del proyecto para la nota final } \\
\text { Más trabajo en grupo } \\
\text { Motivar más a los compañeros para que participen más }\end{array}$ & Está bien como está & No sé \\
\hline
\end{tabular}

g) Las sesiones comenzaron en la segunda evaluación y supusieron la introducción de una nueva modalidad de actividades. La nota media de los grupos se incrementó durante los meses que duró el proyecto, pasando de 5,2 a 6,1 .

\section{CONClusión}

Tras analizar trabajos de otros autores, páginas web y diferentes experiencias de aula, hemos comprobado que existe una importante cantidad de fragmentos de películas y series susceptibles de ser utilizados como recurso didáctico en clase de Matemáticas, la mayoría disponible de forma online. Asimismo, hemos sugerido una clasificación de este tipo de recursos, similar a la que proponen otros autores y basada en el tipo de actividad que se puede generar.

Hemos visto que la teoría de las situaciones didácticas de Brousseau constituye un marco teórico apropiado sobre el que considerar el diseño instruccional de secuencias de aula haciendo uso de fragmentos de películas. Ahora bien, es importante considerar en el análisis de la transposición didáctica el conocimiento matemático puesto en juego en dicho fragmento, y contrastarlo tanto con el saber académico como con el conocimiento a enseñar.

En la experiencia que hemos descrito, los fragmentos de películas se han revelado como una herramienta útil a la hora de introducir conceptos matemáticos, disminuyendo el salto cognitivo necesario, gracias a la información adicional que aporta el contexto. En una de las secuencias mostradas en este artículo como ejemplo, la escena proporciona evidencia suficiente de que se está cometiendo un error de base matemática, lo que restringe las posibilidades de conceptualizar erróneamente la situación y anima a los alumnos a elaborar una interpretación correcta.

Para la realización de este estudio hemos tomado los elementos centrales de la escuela francesa de Didáctica de las Matemáticas, centrándonos sobre todo en la faceta instruccional. Ahora bien, podría haberse planteado desde una perspectiva diferente, tomando como punto de partida supuestos epistemológicos o incluso semióticos. Por lo tanto, nos parece interesante como futura línea de investigación el enfocar el diseño y análisis de las secuencias didácticas desde la visión del marco que proporciona el enfoque ontosemiótico (Godino, 2002), que analice el lenguaje empleado en la escena y las diferentes representaciones del conocimiento en juego. 


\section{REFERENCIAS BIBLIOGRÁFICAS}

Asti, A. y Beltrán Pellicer, P. (2012). 7art Maths: un progetto vincente. XlaTangente: percorsi nella matematica, 35.

Ballesta Pagan, J. y Guardiola Jiménez, P. (2001). El profesorado ante las nuevas tecnologías y los medios de comunicación. Enseñanza \& Teaching: Revista Interuniversitaria de Didáctica, 19, 211-238.

Bartolomé Pina, A. y Mateo Andrés, J. (1983). Utilización del lenguaje audiovisual en la enseñanza de la Estadística. Enseñanza \& Teaching: Revista Interuniversitaria de Didáctica, 1, 183-192.

Billett, S. (1996). Situated learning: Bridging sociocultural and cognitive theorising. Learning and instruction, 6 (3), 263-280. http://dx.doi.org/10.1016/0959-4752(96)00006-0.

Brousseau, G. (1997). Theory of Didactical Situations in Mathematics: Didactique des mathématiques, 1970-1990 (p. 332). Springer.

Chevallard, Y. (1985). La transposition didactique; du savoir savant au savoir enseigné. París: La Pensée Sauvage.

Cornell Department of Mathematics. (2010). Numb3rs Math Activities. Descargado el día 12 de septiembre de 2012. http://www.math.cornell.edu/ numb3rs,

De Pablos Pons, J. (1989). La diégesis cinematográfica y sus implicaciones didácticas. Enseñanza \& Teaching: Revista Interuniversitaria de Didáctica, 7, 9-16.

Devlin, K. y Lorden, G. (2007). The numbers behind Numb3rs. A Plume Book.

Fandiño Pinilla, M. I. (2007). Fractions: conceptual and didactic aspects. Acta Didactica Universitatis Comenianae, 23-45.

Freudenthal, H. (1991). Revisiting Mathematics Education. Kluwer Academic Publishers.

Godino, J. (2002). Un enfoque ontológico y semiótico de la cognición matemática. Recherches en didactique des Mathématiques, 22, 1-32.

Gómez-Chacón, I. M. (2005). Motivar a los alumnos de secundaria para hacer matemáticas. En Matemáticas: PISA en la práctica. Curso de formación de Profesores. Madrid: Ministerio de Educación y Ciencia.

Greenwald, S. J. y Nestler, A. (2010). Mathematics on The Simpsons. Descargado el día 1 de mayo de 2012.

http://mathsci2.appstate.edu/ sig/simpsonsmath/

Kasman, A. (2012). Mathematical Fiction Homepage. Descargado el día 24 de septiembre de 2012.

http://kasmana.people.cofc.edu/MATHFICT/

Knill, O. (2013). Mathematics in Movies. Descargado el día 10 de enero de 2013. http://www.math.harvard.edu/ knill/mathmovies.

Martín, A. y Martín Sierra, M. (2012). Mathsmovies: La web de las matemáticas y el cine. Descargado el día 12 de septiembre de 2012.

http://www.mathsmovies.com.

Meyer, M.; Dekker, T. y Querelle, N. (2001). Context in Mathematics Curricula. Mathematics teaching in the middle school, 6 (9), 522-527.

Mice-T. (2000). Model Instruments for a common evaluation. Descargado el día 12 de septiembre de 2012.

http://www.mice-t.net/mice.htm 
Palmer, L. (1994). It's a Wonderful Life: Using Public Domain Cinema Clips To Teach Affective Objectives and Illustrate Real-World Algebra Applications. ISETA Conference.

Población Sáez, A. J. (2006a). Las matemáticas en el cine. Proyecto Sur de Ediciones S. L. Real Sociedad Matemática Española.

Población Sáez, A. J. (2006b). ¿Las matemáticas en el cine? SCTM06: sociedad, ciencia, tecnología y matemáticas (pp. 1-8).

Polster, B. y Ross, M. (2012). Math Goes to the Movies. Johns Hopkins University Press.

Raga Benedicto, M. C.; Muedra Jornet, A. y Requena Sala, J. L. (2009a). Matemáticas de cine: una propuesta innovadora. Suma: Revista sobre Enseñanza y Aprendizaje de las Matemáticas (62), 19-24.

Raga Benedicto, M. C.; Muedra Jornet, A. y Requena Sala, J. L. (2009b). Matemàtiques de cine. Valencia: Generalitat Valencia.

Requena, Á. (2013). Matemáticas de cine: Recursos para el aula. Descargado el día 12 de enero de 2013.

http://matedecine.wordpress.com,

Roberts, F. y Roberts, D. (2013). Math and the Movies! Descargado el día 12 de enero de 2013.

http://mathbits.com/MathBits/MATHMOVIES/MathMovies.htm.

Sorando Muzás, J. M. (2007). Gazapos matemáticos en el cine y en la televisión. Suma: Revista sobre Enseñanza y Aprendizaje de las Matemáticas, 55, 117-125.

Sorando Muzás, J. M. (2011). De cine. Aventuras y matemáticas. Actividades. Descargado el día 12 de septiembre de 2012.

http://venxmas.fespm.es/temas/de-cine-aventuras-y-matematicas.htm.

Sorando Muzás, J. M. (2012). Matemáticas en tu mundo: Matemáticas en el Cine y en las series de TV. Un recurso para el aula. Descargado el día 12 de septiembre de 2012. http://catedu.es/matematicas_mundo/CINE/cine.htm

Spanhel, D. (2011). Un enfoque para integrar la educación en medios, en la instrucción y en el día a día de la escuela secundaria. Enseñanza \& Teaching: Revista Interuniversitaria de Didáctica, 29, 181-190.

Verret, M. (1975). Le temps des études. París: Librairie Honoré Champion. 